\title{
Pathological Complete Response following Neoadjuvant Chemotherapy in Invasive Ductal Carcinoma with Mammary Paget's Disease: A Case Report
}

\author{
Wataru Goto ${ }^{a}$ Shinichiro Kashiwagi ${ }^{a} \quad$ Yuko Kawano $^{a} \quad$ Asuka Komoda $^{a}$ \\ Sae Ishihara ${ }^{a}$ Yuka Asano $^{a} \quad$ Tamami Morisaki $^{a}$ Kosei Hirakawa ${ }^{a}$ b \\ Masaichi Ohira $a, b$ \\ aDepartment of Breast and Endocrine Surgery, Osaka City University Graduate School of \\ Medicine, Osaka, Japan; b Department of Gastrointestinal Surgery, Osaka City University \\ Graduate School of Medicine, Osaka, Japan
}

\author{
Keywords \\ Paget's disease - Neoadjuvant chemotherapy · Pathological complete response $\cdot$ Breast \\ cancer $\cdot$ Mammary tumor
}

\begin{abstract}
Mammary Paget's disease is a rare malignancy. Mastectomy or breast-conserving surgery has been considered as the standard treatment, while there have been few reports of neoadjuvant chemotherapy (NAC). A 53-year-old woman with erythema and skin ulceration of the left breast was admitted to our institution. Breast examinations revealed left invasive ductal carcinoma (cT1bN0M0, cStage I), and a punch biopsy of the left mammary erythema indicated Paget's disease (cTisNOM0, cStage0). The patient received NAC because of multiple lesions. Consequently, the breast tumor clinically disappeared, and the erythema improved. These outcomes made it easier to perform surgery (left mastectomy and sentinel node biopsy). Histopathological examination revealed no residual cancer cells in either the mammary gland or breast skin, and no metastasis was found in the sentinel lymph node. Therefore, NAC may be a useful treatment for mammary Paget's disease.
\end{abstract}

\section{Karger $\stackrel{2}{=}$}


Goto et al.: NAC for IDC with Mammary Paget's Disease

\section{Introduction}

Mammary Paget's disease is a relatively uncommon type of breast cancer localized in the nipple epidermis, often extending to the lactiferous duct and mammary gland [1]. Paget's disease is characterized by an eczematous eruption and ulceration of the nipple that can affect the areola [2]. These symptoms can negatively affect the patients' quality of life. According to the National Comprehensive Cancer Network (NCCN) guidelines, the main treatments for mammary Paget's disease are mastectomy or breast-conserving surgery followed by radiation therapy. Chemotherapy and endocrine therapy for mammary Paget's disease can be utilized as adjuvant treatments, depending on the clinical situation, such as the presence or absence of invasive areas. In contrast, there have been few reports of neoadjuvant chemotherapy (NAC). We report a rare case of NAC for invasive ductal carcinoma coexisting with mammary Paget's disease, which resulted in a pathological complete response (pCR).

\section{Case Report/Case Presentation}

A 53-year-old woman was admitted to our hospital because of abnormalities in the screening mammography findings. She was unaware of a breast mass but had a history of erythema and skin ulceration with pain and hemorrhage in her left breast. The symptoms started 2 years prior and had progressed. On examination, her skin erythema spread from the left nipple extending outside of the areola, and the area was approximately $8.5 \times 6.5$ $\mathrm{cm}$ in size (Fig. 1a). No breast or axillary lymph nodes were palpable.

Ultrasonography findings showed an irregular, ill-defined hypoechoic lesion at zone D of the left breast, measuring $6.7 \times 6.7 \times 4.3 \mathrm{~mm}$ (Fig. 1b). There were no enlarged axillary lymph nodes. CT findings showed no lymph nodes or distant metastases (Fig. 1c). MRI revealed an approximately 7 -mm irregular tumor in the region of the left mammary gland and focal skin thickening with enhancement around the left nipple-areolar complex (Fig. 1d).

Core needle biopsy of the left breast tumor indicated an invasive ductal carcinoma. Immunohistochemical examination revealed that the tumor was estrogen receptor negative, progesterone receptor negative, and human epidermal growth factor receptor 2 (HER2) positive. A punch biopsy of the left mammary erythema revealed proliferation of characteristic Paget cells within the epidermis. These atypical cells were large, pale, and had abundant clear cytoplasm, and immunohistochemistry showed a high expression of HER2 (Fig. 2a, b).

The pretreatment diagnosis was left invasive ductal carcinoma (cT1bN0M0, cStage I, HER2 enriched) coexisting with Paget's disease (cTisN0M0, cStage0). Because of the HER2enriched subtype of invasive cancer, she received NAC consisting of 4 courses of paclitaxel, trastuzumab, and pertuzumab. A repeat breast examination, ultrasonography, CT, and MRI after NAC showed absence of tumor in the left mammary gland and improvement of skin thickening (Fig. 3a-d). The preoperative diagnosis was left breast cancer (ycTisN0M0, ycStage I) and Paget's disease (ycTisN0M0, ycStage 0), and she underwent left mastectomy and sentinel node biopsy. In addition, because the skin defect after mastectomy was large and the direct closure was difficult to cover, reconstructive surgery using a rhomboid flap was performed. Histopathological examination revealed no residual cancer cells in either the breast tumor or Paget's disease, and no metastasis in the sentinel lymph node (pCR) was noted.

\section{Karger'}



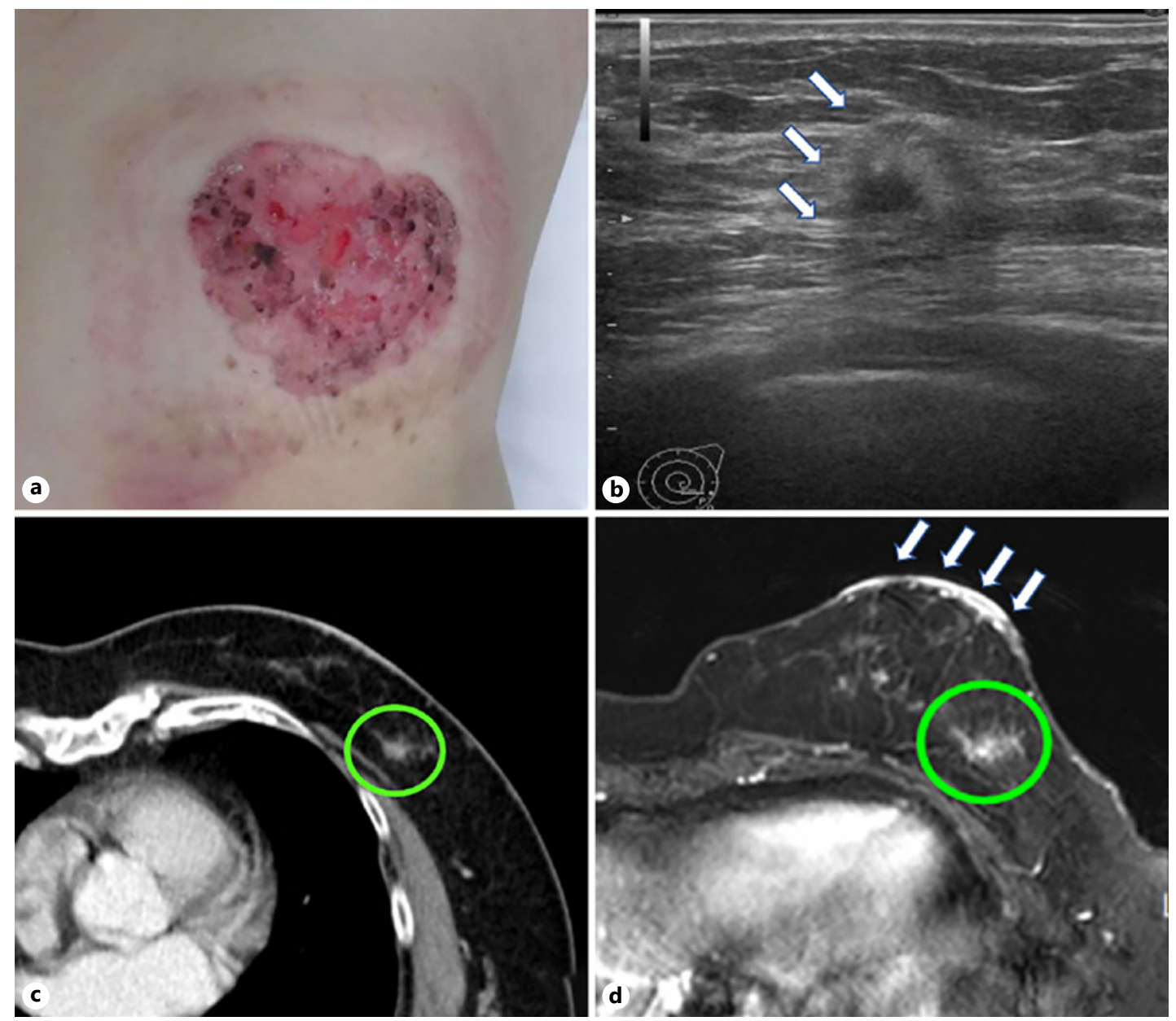

Fig. 1. a Pretreatment findings. Macroscopic view of the left breast. The area of the erythema with skin ulceration was $8.5 \times 6.5 \mathrm{~cm}$. b Breast ultrasound revealed a $6.7 \times 6.7 \times 4.3 \mathrm{~mm}$-sized, irregular, ill-defined hypoechoic lesion at zone D of the left breast (arrow). c CT showed the tumor in the left mammary gland (circle). d MRI revealed an irregular tumor in a region of the left mammary gland (circle) and focal skin thickening with enhancement around the left nipple-areolar complex (arrow).

\section{Discussion/Conclusion}

We report a rare case of breast cancer with mammary Paget's disease treated with NAC and achieved pCR. There are 2 different theories in the pathogenesis of mammary Paget's disease: first, the malignant cells of noninvasive carcinoma just below the nipple migrate through the milk ducts along the epidermis of the areola (epidermotropic theory) [3], and second, the epidermal cells of the nipple undergo a malignant transformation in situ (intraepidermal transformation theory) [4]. The first theory is widely accepted because most studies report the presence of concurrent underlying breast cancer. In our case, tumors in the mammary gland and Paget cells within the epidermis shared the same immunohistochemical characteristics: a high expression of HER2. However, the MRI showed no obvious intraductal extension of the breast cancer, and a punch biopsy of the skin indicated no stromal invasion of malignant cells; hence, we consider that the Paget's disease was caused by the second theory. 


\section{Case Reports in Oncology}
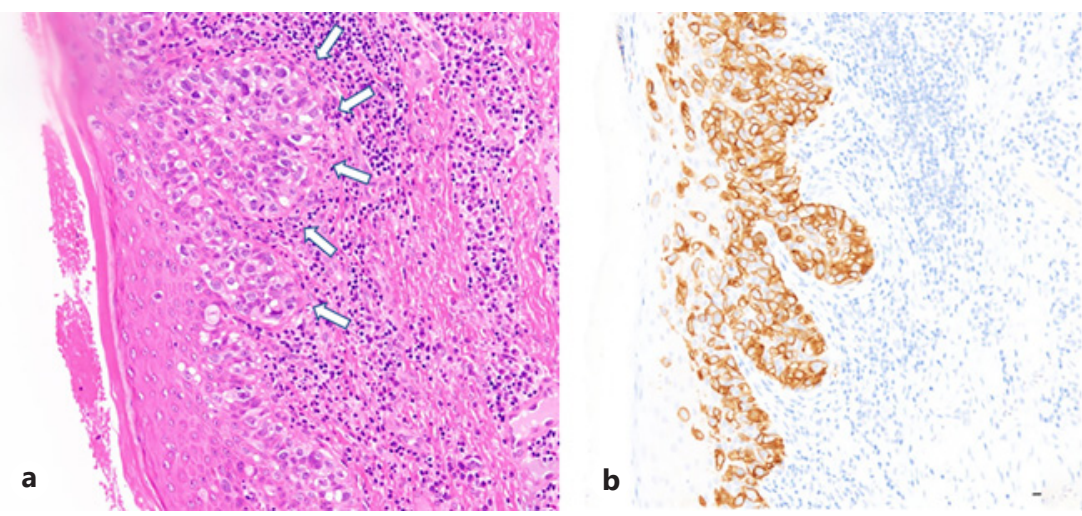

Fig. 2. Pathological examination of punch biopsy of left mammary erythema. Characteristic Paget cells were detected within the epidermis (arrow). a No infiltration was observed. b Immunohistochemistry for HER2 showed high expression on the membrane of the Paget cells. HER2, human epidermal growth factor receptor 2 .
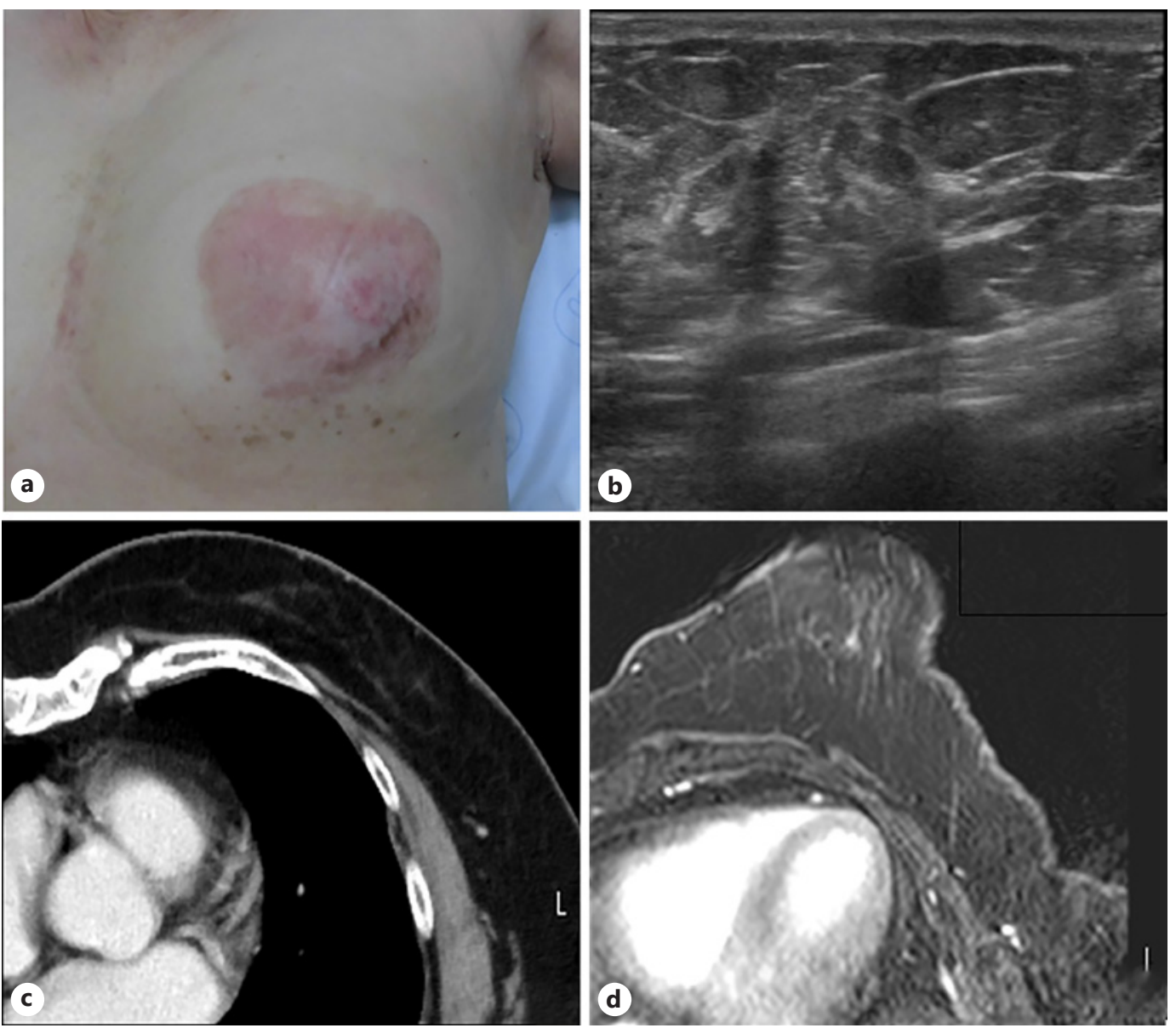

Fig. 3. Preoperative findings. a The erythema of the left breast was improved after NAC. b-d Ultrasound, CT, and MRI after NAC showed no tumor in the left mammary gland and improvement of skin thickening. NAC, neoadjuvant chemotherapy. 
Mammary Paget's disease generally requires mastectomy or breast-conserving surgery followed by radiation therapy [5]. In our case, because mammary Paget's disease was accompanied by invasive ductal carcinoma, we performed treatment for invasive breast cancer based on the NCCN guidelines. In our institution, HER2-postive breast cancer larger than $10 \mathrm{~mm}$ is treated with NAC, while breast cancer between 6 and $9 \mathrm{~mm}$ (T1b) is considered for NAC, depending on the risk of recurrence. The present case had multiple lesions (Paget's disease plus invasive ductal carcinoma), and we performed NAC and achieved pCR. Although adjuvant chemotherapy or endocrine therapy for mammary Paget's disease may be considered depending on the staging of the underlying carcinoma, NAC is not a standard treatment [6]. However, in our case, NAC was effective in reducing the size of the erythema caused by the Paget's disease. Mammary Paget's disease sometimes requires resection of a large area, and skin graft techniques, such as myocutaneous flaps, are often used to cover such defects. However, they require more time and involve more blood loss. In this case, since the erythema was reduced by NAC, we were able to choose a reconstruction technique (rhomboid flap) with less blood loss and a shorter operation time. According to the above results, NAC may be a useful treatment for giant mammary Paget's disease.

In invasive breast cancer, pCR after NAC is an independent predictor of good outcomes in HER2-enriched and triple-negative breast cancer subtypes [7, 8]. However, the relationship between pCR and prognosis of mammary Paget's disease is unclear, and further studies are needed.

\section{Acknowledgment}

We thank Yayoi Matsukiyo and Tomomi Okawa (Department of Breast and Endocrine Surgery, Osaka City University Graduate School of Medicine) for helpful advice regarding data management.

\section{Statement of Ethics}

Written informed consent for the publication of the present case report was obtained from the patient.

\section{Conflict of Interest Statement}

The authors declare that they have no conflicts of interest to disclose.

\section{Funding Sources}

The authors did not receive any funding.

\section{Author Contributions}

W.G. contributed to writing of this manuscript. S.K. and Y.K. supervised the treatment plans. W.G., S.K., and Y.K. performed the chemotherapy and operation. A.K., S.I., Y.A., and T.M. summarized the data. K.H. and M.O. made substantial contribution to the study design and performed the treatment. All authors read and approved the final edition of this manuscript.

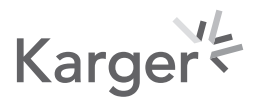




\section{Case Reports in Oncology}

\begin{tabular}{l|l}
\hline Case Rep Oncol 2021;14:1242-1247 \\
\hline DOI: 10.1159/000516762 & $\begin{array}{l}\text { @ 2021 The Author(s). Published by S. Karger AG, Basel } \\
\text { www.karger.com/cro }\end{array}$ \\
\hline
\end{tabular}

Goto et al.: NAC for IDC with Mammary Paget's Disease

\section{References}

1 Sandoval-Leon AC, Drews-Elger K, Gomez-Fernandez CR, Yepes MM, Lippman ME. Paget's disease of the nipple. Breast Cancer Res Treat. 2013 Aug;141(1):1-12.

2 Lohsiriwat V, Martella S, Rietjens M, Botteri E, Rotmensz N, Mastropasqua MG, et al. Paget's disease as a local recurrence after nipple-sparing mastectomy: clinical presentation, treatment, outcome, and risk factor analysis. Ann Surg Oncol. 2012 Jun;19(6):1850-5.

3 Paone JF, Baker RR. Pathogenesis and treatment of Paget's disease of the breast. Cancer. 1981 Aug 1;48(3): 825-9.

4 Inglis K. Paget's disease of the nipple; with special reference to the changes in the ducts. Am J Pathol. 1946 Jan; 22:1-33.

5 Lim HS, Jeong SJ, Lee JS, Park MH, Kim JW, Shin SS, et al. Paget disease of the breast: mammographic, US, and MR imaging findings with pathologic correlation. Radiographics. 2011 Nov-Dec;31(7):1973-87.

6 Kawase K, Dimaio DJ, Tucker SL, Buchholz TA, Ross MI, Feig BW, et al. Paget's disease of the breast: there is a role for breast-conserving therapy. Ann Surg Oncol. 2005 May;12(5):391-7.

7 Bonnefoi H, Litière S, Piccart M, MacGrogan G, Fumoleau P, Brain E, et al. Pathological complete response after neoadjuvant chemotherapy is an independent predictive factor irrespective of simplified breast cancer intrinsic subtypes: a landmark and two-step approach analyses from the EORTC 10994/BIG 1-00 phase III trial. Ann Oncol. 2014 Jun;25(6):1128-36.

8 Kuroi K, Toi M, Ohno S, Nakamura S, Iwata H, Masuda N, et al. Prognostic significance of subtype and pathologic response in operable breast cancer; a pooled analysis of prospective neoadjuvant studies of JBCRG. Breast Cancer. 2015 Sep;22(5):486-95. 